\title{
一种基于复杂系统观的效能分析新方法: 单调指标空间分析方法
}

\author{
胡剑文 ${ }^{1,2^{* *}}$ 张维明 ${ }^{1}$ 胡晓峰 ${ }^{2}$ 刘 忠 ${ }^{1}$ 司光亚 $^{2}$
}

(1. 国防科技大学 C4ISR 技术重点实验室, 长沙 410073; 2. 国防大学战略模拟教研室, 北京 100091)

\begin{abstract}
摘要 根据复杂系统的整体性、非线性、不确定性、开放性等特点, 提出了一 种新的效能分析方法：单调指标空间分析方法，并应用其于一反隐身防空信息系 统的效能分析. 论文首先提出了指标单调性公设, 单调指标空间, 单调指标需求 轨迹, 总单调指标需求轨迹, 模糊单调指标需求轨迹等概念. 然后提出了单调指 标需求轨迹生成的一种并行数值算法. 算法根据单调性公设, 采取了超盒数值逼 近的方法, 快速逼近所求的系统单调指标需求轨迹, 并通过模拟令牌环机制实现 了算法的并行处理。此算法极大地缩减了搜索空间, 很大程度上克服了依赖显式 解析函数关系求单调指标需求轨迹的缺陷. 然后, 根据实际应用的需求, 提出了 具有各自优缺点以及适用范围的三种单调指标需求轨迹求交的方法, 它们是: 直 接求交法, 逼近法, 优化蒙特卡罗方法. 第三, 在前面研究的基础上, 提出了基 于单调指标需求轨迹的系统分析模型, 它们是系统的有效性评估模型, 指标的灵 敏度分析模型, 模糊效能分析模型. 最后, 运用前述理论方法于一反隐身防空信 息系统的分析，显示出了本方法具有良好的应用前景.
\end{abstract}

\section{关键词 单调指标空间 单调指标需求轨迹 效能分析 模糊效能分析}

\section{1 引言}

系统的效能是一个系统满足一组特定任务要求程度的度量. 在自然科学与 社会科学中的很多领域都涉及到复杂系统的效能分析问题, 它是一个非常困难 而很有价值的研究课题. 多年来它一直是学术界的热点问题, 因此出现了许多有 影响的效能分析方法. 例如:

2004-11-10 收稿, 2005-03-01 收修改稿

* 国家自然科学基金资助项目 (批准号: 70401003)

** E-mail: hjwc3i@sina.com 
指数法, 它具有综合能力强, 方法直观等优点; 但其分析过程受人为主观因 素影响大(如关于“权”值的确定等), 效能分析结果的涵义不够明确.

美国工业界武器系统效能咨询委员会提供的效能评价 ADC 模型 ${ }^{[1]}$, 它是以 系统状态划分及其条件转移概率为建模思想的, 其数学过程严格, 所获得的效能 分析结果涵义明确; 但它应用于系统状态数较多的复杂系统时, 会出现矩阵维数 成指数规律增长.

基于加权综合多属性决策的效能分析方法 [2]. 其中比较著明的有: 层次分析 法, ELECTRE 方法, LINAMP 方法等等，这些方法通过各种方式确定指标的权值， 然后加权综合, 以进行效能分析; 但是简单地线性综合各个分指标以进行系统的 整体效能分析是违背复杂系统整体性特征的，其有效性受很大的限制，另外分析 的结果涵义也不明确.

SEA方法 ${ }^{[3,4]}$, 它通过把系统能力和使命要求在同一公共属性空间内进行比 较, 得到有效性的若干度量, 在此基础上适当地组合这些分度量, 便可以得到系 统的总度量. 这是一种较好体现系统整体特性的方法, 但是系统的使命要求难以 有效确定，这是 SEA 方法的最大缺陷.

本文根据复杂系统的整体性、非线性、不确定性、开放性等特点，提出了一 种新的效能分析方法: 单调指标空间分析方法, 它能在一定程度上弥补上述方法 的部分缺陷. 最后应用其于一反隐身防空信息系统的效能分析, 显示出其具有良 好的应用前景.

\section{2 复杂系统特性 ${ }^{[5,6]}$ 及其对系统效能分析的启示}

\section{1 整体性}

复杂系统的整体性要求在效能分析时, 不应用各部分简单的组合以替代整 体, 即系统的总效能值不能够用各个分指标的简单组合(如线性加权等). 对于复 杂系统来说各个指标存在紧密的相关性, 彼此间不可能效用独立, 是不能用加性 或拟加性函数来表示整体效用的.

\section{2 非线性}

复杂系统是难以用基于线性关系的数学模型甚至是一般的解析模型来建模 的, 难以在系统性能指标(或效能指标)与使命需求指标之间建立显式的函数关系.

\section{3 开放性}

由于系统实际效能只能在具体的环境下体现, 因此, 系统效能具有强烈的相 对特性, 不同的环境下系统有效性可能不一样, 进行系统效能分析时, 应综合考 虑各种典型外部环境. 


\section{4 不确定性}

系统效能(性能)指标存在不确定性(随机与模糊的). 产生不确定的原因是由 于系统运行的随机漂移而产生的系统运行状态多值性与随机性分布; 另外, 系统 运行状态与系统所处的环境密切相关, 而系统的环境又是多变的, 这些都是造成 系统不确定的重要原因.

效能分析方法应该建立在复杂系统基本特性的基础上, 这样才能反应系统 的本质, 效能分析方法也应建立在复杂系统观之上. 本文提出的效能分析方法基 本遵循着复杂系统的根本特性.

对于系统的整体性，本文借鉴了 SEA 方法的分析思想，整体地分析各个分指 标，而不是把它们简单地加权综合.

针对系统的非线性特性, 本文提出了一个基于数值关系模型的单调指标需 求轨迹快速生成算法, 它相对于文献[3,7,8]中采用线性微分方程的方式来建立需 求指标与系统指标的映射关系的方式，更适于复杂系统.

针对系统的开放性，本文的方法把系统放在典型的运作环境中来分析，综合 考虑各种运作环境下的系统效能.

针对系统的不确定性, 我们运用概率密度函数来描述系统的指标. 另外, 针 对需求描述的模糊性, 运用了模糊理论(如模糊积分方法)来进行系统的效能分析.

\section{3 单调指标空间中的公设与基本概念}

公设: 系统效能(性能)指标与使命需求指标之间具有单调关系

本文把系统效能(性能)指标与使命需求指标之间具有单调关系作为一个公设, 这种单调性是普遍的. 例如: 在文献[3]中, 显然对于可靠性 $R$, 生存能力 $S$, 反应 时延 $T$, 与允许流量 $K$, 这几个指标相对使命需求指标敌我损失比, 显然是单调 的; 在文献[4]中, 链路系统可靠性与反应时延相对于杀伤概率也是单调的; 在文 献[7 9]中, 它们系统(效能, 性能)指标相对于需求指标都是单调的. 把单调性作 为一个公设是合理的.

定义 1 单调指标空间 $P$. 设 $P^{n}$ 为 $n$ 维欧几里德空间, 设 $P \subset R^{n}, P$ 是系统 效能(性能)指标点的集合, 每一维都代表了一项指标. 并存在 $f_{u}, f_{u}$ 是定义在 $P$ 上的一连续区域，表示效能(或性能)指标与使命需求指标 $u$ 的函数关系. 即: $f_{u}$ : $P \rightarrow u$. 它是 $n$ 元单调函数, 即某个指标在其它各个指标的值确定时, $f_{u}$ 就是普通 的一元单调函数.

单调指标空间也就是由 $n$ 个指标有效区域所合成的 $n$ 维欧氏空间的子集. 例 如: 对一防空导弹系统, 我们取搜索雷达探测性能指标 $p_{1} \in P_{1}$, 系统延延迟指标 $p_{2} \in P_{2}$, 跟踪雷达跟踪性能指标 $p_{3} \in P_{3}$ 等几个关键指标, 单调指标空间 $P$ 就是 
这三个指标的笛卡尔积: $P=P_{1} \times P_{2 \times} \times P_{3}$. 可以建立三元函数 $u=f_{u}\left(p_{1}, p_{2}, p_{3}\right)$, 其表示 三个指标到毁敌概率 $u$ 的函数关系. 由于敌情和环境的多样性(例如: 来袭目标具 有不同的类型 $), f_{u}\left(p_{1}, p_{2}, p_{3}\right)$ 可以分解成多个函数 $f_{u i}\left(p_{1}, p_{2}, p_{3}\right), i=1 . . m$. 比如用 $f_{u 1}$ $\left(p_{1}, p_{2}, p_{3}\right)$ 表示来袭目标是隐身飞机时, 三个指标到毁敌概率的函数关系; $f_{u 2}\left(p_{1}\right.$, $p_{2}, p_{3}$ ) 为巡航导弹时等等. 很显然 $f_{u}$ 具有单调性. 相对于毁敌概率, 搜索雷达探测 性能指标 $p_{1}$ 是单调增的, 目标指示迟延 $p_{2}$ 是单调减的, 跟踪雷达跟踪性能指标 $p_{3}$ 是单调增的.

为了表示的简洁性, 可以通过简单的转化把单调减的指标变成单调增的形 式, 例如, 上述 $p_{2}$ 是单调减的类型, 如果取一个较大的数 $T$, 用 $T-p_{2}$ 代替 $p_{2}$, 即 可化成单调增的形式.

定义 2 单调指标需求轨迹 $P l$ 设 $P l \subseteq P$, 给定一使命需求指标值 $u_{k}$, $\forall x \in P l, f_{u}(x) \leqslant$ 或 $\geqslant u_{k}$ ( “ $\leqslant$ 或 $\geqslant ”$ 和 $u_{k}$ 根据实际需求确定 $)$, 则 $P l$ 称为单调指标需 求轨迹.

轨迹(locus)是指: 坐标满足某一等式或一个或多个代数条件的所有的点的集 合或组合. 单调指标需求轨迹也就是在单调指标空间中能够满足某项需求的指 标点. 如上例中, 用户要求击毁敌隐身飞机概率大于等于 0.6 , 则在 $P$ 中所有满足 $f_{u}\left(\mathrm{p}_{1}, p_{2}, p_{3}\right) \geqslant 0.6$ 点的集合为 $P l$.

定义 3 总单调指标需求轨迹 $T P l, T P l=\bigcap_{i} P l_{i} . P l_{i}$ 是针对第 $i$ 项使命需求所 对应的单调指标需求轨迹. $T P l$ 即为 $P$ 中能满足用户各项需求的子集.

定义 4 模糊单调指标需求轨迹 $f p l(\lambda)$. 在一定的论域上建立使命的模糊集 $\tilde{R}$, 令 $\tilde{R}_{\lambda}$ 为模糊集 $\tilde{R}_{\lambda}$ 的 $\lambda$ 集, 模糊单调指标需求轨迹定义为单调指标空间 $P$ 中 能够达到 $\tilde{R}_{\lambda}$ 使命需求的区域, 记为 $f p l(\lambda)$.

现举一例说明模糊单调指标需求轨迹的含义. 如果对某一防空系统, 其使命 需求指标为模糊集击落敌机 $R$, 论域为击落敌机的概率值. 对于给定的一个隶属 值 $\lambda$ 都可求出 $R_{\lambda}$. $R_{\lambda}$ 即为对击落敌机的概率使命约束(即必须大于某一最小概率 值), 进一步可得到基于 $\lambda$ 的单调指标需求轨迹 $f p l(\lambda)$. 显然根据模糊集的分解定 理, 当 $\lambda_{1} \leqslant \lambda_{2}$ 时, $\tilde{R}_{\lambda_{1}} \supseteq \tilde{R}_{\lambda_{2}}$, 再根据单调性公设则 $f p l\left(\lambda_{1}\right) \supseteq f p l\left(\lambda_{2}\right)$.

\section{4 单调指标空间中的单调指标需求轨迹生成算法}

\section{1 有关的定义与定理}

单调指标需求轨迹是进行系统效能分析的基础. 所以, 进行系统效能分析的 前提与关键就是求出单调指标需求轨迹. 根据定义 2 , 求出单调指标需求轨迹的 关键也就是确定 $f_{u}$. 目前, 它从表示形式上可分成以下五种类型: 
1. 显式函数(包括可以得出解析式的线性微分方程或方程组);

2. 一种隐函数, 例如无法求出解析解的非线性微分方程(组);

3. 数据表格;

4. 仿真试验;

5. 数学规划;

第一种形式是最理想的形式. 可直接得出系统的单调指标需求轨迹解析形 式. 例如: 文献[3]在进行某防空 $\mathrm{C}^{3} \mathrm{I}$ 系统进行有效性分析时，使用了一个齐次火 力的 Lanchester 作战模型来实现这种映射. 但是, 用一个普通的线性 Lanchester 作战模型来描述战争是一种简化论的观点，其有效性极低，不符合复杂系统的特 性. 这类模型的确不适合于对抗条件下的描述，对于非对抗条件其具有一定的适 用性. 后四种形式适于描述复杂系统，但其无法得到相应的解析式，无法直接导 出单调指标需求轨迹. 另外, 即使能够用第一种形式描述, 也常常由于积分计算, 集合运算等要求, 也需要利用数值方法把单调指标需求轨迹逼近分割成互不相 交规则区域的集合. 因此，必须研究基于数值逼近的单调指标需求轨迹的生成 方法.

效能地需求轨迹的生成就是基于相应 $f_{u}$ 与使命需求指标的约束，如何在单调 指标空间中寻找符合需求的指标点. 如前面所述一般情况下是难以得出显示函 数形式的, 下面主要讨论在无显式数的情况下, 如何用数值方法逼近相应的单调 指标需求轨迹.

为了简明地表示问题，不妨设指标都为单调增的(对于单调减类型的指标按 上述方法作转换处理即可), 求出满足需求 $f_{u}\left(p_{1}, p_{2}, \cdots, p_{n}\right) \leqslant u_{k}, u_{k} \geqslant 0$ 的相应 单调指标需求轨迹, 对于 $f_{u}\left(p_{1}, p_{2}, \cdots, p_{n}\right) \geqslant u_{k}$ 类型的处理同理. 并把各个指标 $p_{i}$ 规范到区间 $\left[0, V_{i}\right]$ 内.

定理 1 单调指标需求轨迹的基本性质定理当 $P l$ 非空时, 单调指标需求轨迹 是连通的空间区域, 即 $P l$ 中的任意两点都可以在 $P l$ 中找到连续路径连接.

证 设 $\forall A, B \in P l, A$ 点到 0 点多维直线可以表示为 $L_{A}=\{p \mid 0 \leqslant k \leqslant 1$, $\left.p=\left(k V_{A 1}, k V_{A 2}, \cdots, k V_{A n}\right)\right\}$, 其中 $V_{A i}$ 为 $A$ 点的第 $i$ 个坐标值, 当 $A \in P l$ 时, 根据单 调性 $L_{A} \subset P l$. 同理 $L_{B} \subset P l$, 由于 $f_{u}$ 的定义域是连续的, 因此 $P l$ 中的任意两点都 可用两条包含在 $P l$ 中的多维直线连通. 不存在孤立的两个点, 也即 $P l$ 是个整体.

定义 5 最小外包超盒 单调指标需求轨迹中的各个指标取值的上下确界的 笛卡尔积. 即: $\left[0, V_{1}^{\prime}\right] \times\left[0, V_{2}^{\prime}\right] \times \cdots \times\left[0, V_{n}^{\prime}\right]$, 其中 $V_{i}^{\prime} \leqslant V_{i}$.

例如: 假设 $x, y$ 是指标, 满足式 $x^{2}+y^{2} \leqslant 4(x \geqslant 0, y \geqslant 0)$ 的点集为单调指 标需求轨迹，则区域 $[0,2] \times[0,2]$ 就是最小外包超盒. 最小外包超盒内任一点不一 
定在单调指标需求轨迹内, 但是最小外包超盒外的任何一点都不在单调指标需 求轨迹内.

定义 6 最大等分内接超盒及对角超盒 设 $\left[0, V_{1}^{\prime}\right] \times\left[0, V_{2}^{\prime}\right] \times \cdots \times\left[0, V_{n}^{\prime}\right]$ 为最小 外包超盒. 取一比例系数 $K \in[0,1]$, 令 $\omega=\left[0, K V_{1}^{\prime}\right] \times\left[0, K V_{2}^{\prime}\right] \times \cdots \times\left[0, K V_{n}^{\prime}\right], \omega \subseteq P l$, 且 对 $\forall K^{\prime}>K,\left[0, K^{\prime} V_{1}^{\prime}\right] \times\left[0, K^{\prime} V_{2}^{\prime}\right] \times \cdots \times\left[0, K^{\prime} V_{n}^{\prime}\right] \not \subset P l$, 则定义 $\omega$ 为最大等分内接超 盒. 定义 $\bar{\omega}=\left(K V_{1}^{\prime}, V_{1}^{\prime}\right] \times\left(K V_{2}^{\prime}, V_{2}^{\prime}\right] \times \cdots \times\left(K^{\prime} V_{n}^{\prime}, V_{n}^{\prime}\right]$ 为对角超盒.

上面定义中所谓等分是指用相同的 $K$ 等比分割各个指标，例如对于上例 $x^{2}+$ $y^{2} \leqslant 4(x \geqslant 0, y \geqslant 0)$; 区域 $[0,2] \times[0,2]$ 是最小外包超盒, 而 $[0, \sqrt{2}] \times[0, \sqrt{2}]$ 是最大等分内接超盒, $(\sqrt{2}, 2] \times(\sqrt{2}, 2]$ 为其对角超盒. 其中 $K=\sqrt{2} / 2$.

定理 2 最大等分内接超盒的对角超盒中的任一点都不符合系统需求约束条 件，即不在单调指标需求轨迹内.

证 令 $\omega=\left[0, K V_{1}^{\prime}\right] \times\left[0, K V_{1}\right] \times \cdots \times\left[0, K V_{2}^{\prime}\right]$ (最大等分内接超盒)

假设 $\exists p \in \bar{\omega}=\left(K V_{1}^{\prime}, V_{1}^{\prime}\right] \times\left(K V_{2}^{\prime}, V_{2}^{\prime}\right] \times \cdots \times\left(K V_{n}^{\prime}, V_{n}^{\prime}\right], p=\left(p_{1}, p_{2}, \cdots, p_{n}\right)$, 且 $p \in P l$, 即在效能需求轨迹内.

令 $K p=\min \left(\frac{p_{1}-K V_{1}^{\prime}}{V_{1}^{\prime}}, \frac{p_{2}-K V_{2}^{\prime}}{V_{2}^{\prime}}, \cdots, \frac{p_{n}-K V_{n}^{\prime}}{V_{n}^{\prime}}\right)$, 根据定义 $6, K p>0$.

很显然, 对 $\forall j,(K p+K) V_{j}^{\prime} \leqslant\left(\frac{p_{j}-K V_{j}^{\prime}}{V_{j}^{\prime}}+K\right) V_{j}^{\prime}=p_{j}$,

再令 $p_{k}=\left((K p+K) V_{1}^{\prime},(K p+K) V_{2}^{\prime} \cdots(K p+K) V_{n}^{\prime}\right)$, 根据 $f_{u}$ (定义见上文)单调性, $f_{u}\left((K p+K) V_{1}^{\prime},(K p+K) V_{2}^{\prime}, \cdots,(K p+K) V_{n}^{\prime}\right) \leqslant f_{u}\left(p_{1}, p_{2}, \cdots, p_{n}\right)$.

又因为 $p \in P l$, 所以 $p_{k} \in P l$,

又根据单调性对 $\forall p^{\prime} \in\left[0,(K p+K) V_{1}^{\prime}\right] \times\left[0,(K p+K) V_{2}^{\prime}\right] \times \cdots \times\left[0,(K p+K) V_{n}^{\prime}\right]$

$f_{u}\left(p_{1}^{\prime}, p_{2}^{\prime}, \cdots, p_{n}^{\prime}\right) \leqslant f_{u}\left(p_{k 1}, p_{k 2}, \cdots, p_{k n}\right)$, 所以

$\left[0,(K p+K) V_{1}^{\prime}\right] \times\left[0,(K p+K) V_{2}^{\prime}\right] \times \cdots \times\left[0,(K p+K) V_{n}^{\prime}\right] \subseteq P l$ (其与 $P l$ 凹凸特性无 关), 由于 $K p+K>K$

这与 $\omega=\left[0, K V_{1}^{\prime}\right] \times\left[0, K V_{1}^{\prime}\right] \times \cdots \times\left[0, K V_{2}^{\prime}\right]$ 是最大等分内接超盒矛盾. 假设不 成立.

\section{2 单调指标需求轨迹生成算法}

本算法以递归的方式利用最大等分内接超盒按任意精度来逼近单调指标需 
求轨迹. 其基本步骤如下:

1 确定相应需求约束值 $u_{k}$, 单调指标需求轨迹的生成也即是生成 $P l$, 使 $f_{u}(P l) \leqslant u_{k}$.

2 先求出相应的最小外包超盒. 其求解方法是:

$2.1 k=1$

2.2 把除第 $k$ 个之外所有指标值取 0 (最小值). 根据 $f_{u}$ 的单调性, 可用二分 法求出第 $k$ 个指标的上确界, 它相当于其他指标为最优值时, 第 $k$ 个指标能取的 最差值.

2.3 如果 $k=n$, 各个指标求出的新的区间的笛卡尔积就是最小外包超盒, 退出转 3 . 否则 $k=k+1$, 转 2.2 .

3 求最大等分内接超盒

3.1 判断最小外包超盒的体积(各个区间长度的乘积, 由于量纲不一样要进 行归一化)是否小于某一精度值 $E$. 如果小于则进行适当插值, 然后退出. 另外, 对于某一个分量已小于最小分辨值时, 则此量无须再分解, 因此达到降维的目 的.

3.2 显然根据 $f_{u}$ 单调性, 当 $K_{1} \geqslant K_{2}$ 时, $f_{u}\left(K_{1} V_{1}^{\prime}, K_{1} V_{2}^{\prime}, \cdots, K_{1} V_{n}^{\prime}\right) \geqslant f_{u}\left(K_{2} V_{1}^{\prime}\right.$, $K_{2} V_{2}^{\prime}, \cdots, K_{2} V_{n}^{\prime}$ ), 则同样可用二分法(也可用它的快速逼近方法)试探取值求出 $K$ (最大等分内超盒的分割比例系数), 从而得到最大等分内接超盒.

3.3 最大等分内接超盒的分割比例系数 $K$ 可以把每个指标的在最小外包超 盒内的区间都可分成属于最大等分内接超盒和不属于的两部分. 把属于部分记 为“ “0”, 不属于的记为“ “ ”. 这样就存在 $2^{n}$ ( $n$ 是所有指标的总数)个区间组合, 依次 对应于 0 到 $2^{n}-1$ 的二进制数. 第 0 个, 就是已求出的最大等分内接超盒, 其中任 何一个元素都符合系统需求约束, 无需再分. 而第 $2^{n}-1$ 个即是相应对角超盒. 根 据定理 2, 其中任何一个元素都不符合系统需求约束, 因此去掉. 对剩下的第 1 到 $2^{n}-2$ 区间组合, 依次作为新的最小外包超盒, 递归调用 3. 最后所有最大等分内 接超盒的并集就是系统的单调指标需求轨迹.

算法采用了数值逼近的方法, 逼近整个单调指标需求轨迹空间, 它无需系统 需求与指标间具有精确的函数表达式. 在绝大多数的实际系统中, 很难用普通的 函数来完全表达需求与指标间的关系. 它们之间的关系往往只能用数值仿真模 型来表达, 这类模型只能求出数值解. 本算法使得建立在数值关系模型基础上的 系统单调指标需求轨迹的求解成为可能. 另外, 通过使用规则的超盒来逼近不规 则的单调指标需求轨迹也为后续的分析计算(如, 在单调指标需求轨迹上进行积 分运算, 对单调指标需求轨迹进行集合运算等等)奠定了基础. 第三, 算法还具有 自适应性, 它能够根据单调指标需求轨迹的形状, 以确定逼近超盒的疏密, 通常 
在曲率大的(变化剧烈)地方其超盒数多, 反之则少.

在此，我们用一最简单的例子描述一下算法基本思想，如图 1. 假设单调指 标需求轨迹就是 $x^{2}+y^{2} \leqslant r^{2} ; x \geqslant 0, y \geqslant 0$. 图中虚线边矩形代表最小外包超盒(二维 超盒就是矩形), 实线矩形表示最大等分内接超盒, 根据单调性, 用二分法沿着虚 线箭头方向来回试探, 求出相应的交点(对于连续变量, 只求出近似值), 得出最 大等分内接超盒, 然后去掉对角超盒, 从图中看是显然的, 定理 2 作了严格证明. 生成新的最小外包超盒组后, 再进一步递归 分解. 这样, 所有生成的最大等分内接超盒 的并集就可逼近整个单调指标需求轨迹(圆 的正轴部分).

\section{3 并行实现策略}

我们利用算法的优良模块化特性, 提出 了并行计算的实现方案. 它通过模拟令牌环 机制实现并行处理. 由于篇幅有限, 本文不 再赘述, 可见胡剑文用着色 PETRI 网详细地 描述了整个并行处理的过程 ${ }^{1)}$.

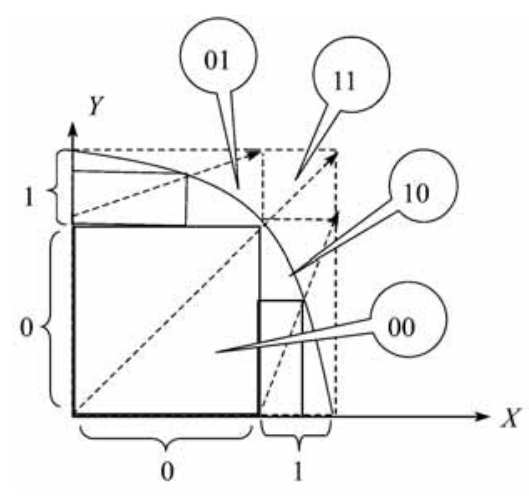

图 1 超盒逼近法求部分圆

\section{5 多单调指标需求轨迹的交运算算法}

在实际问题中, 我们还需要求多个单调指标需求轨迹的交集, 其实际意义也 是显然的. 例如, 某一防空系统可能遇到多样的作战环境, 其使命需求也可能是 多样的, 对系统的最终需求将是所有需求的交集. 另外对敌人伤亡数与自己伤亡 数都是两个使命需求指标, 只有都满足了才能说达到了需求. 我们可以根据较为 典型的几种情况, 求出它们各自的单调指标需求轨迹, 然后作交运算得出总单调 指标需求轨迹. 以下介绍三种不同的求交算法.

\section{1 超盒直接求交法}

由前文可知, 单调指标需求轨迹是由最大等分内接超盒并成的不规则区域, 若对其进行求交运算, 可以直接对规则的超盒求交即可. 这种方法实现起来较为 简单, 但其计算量较大, 例如: 如果两个单调指标需求轨迹进行交运算, 它们各 自由 $M, N$ 个超盒组成, 则须做 $M \times N$ 次超盒求交运算, 才能完成两个单调指标需 求轨迹的交运算. 算法的时间复杂度为 $O\left(n^{2}\right), n$ 表示超盒的个数.

但是, 我们可以很自然地把上节单调指标需求轨迹生成算法所产生的最小 外包超盒与最大等分内接超盒用一个多叉树组织起来, 如图 2 所示,很显然这棵

1) 胡剑文. 复杂系统效能分析中的单调效能空间方法及其应用研究. 国防科技大学博士论文, 2003, 10 


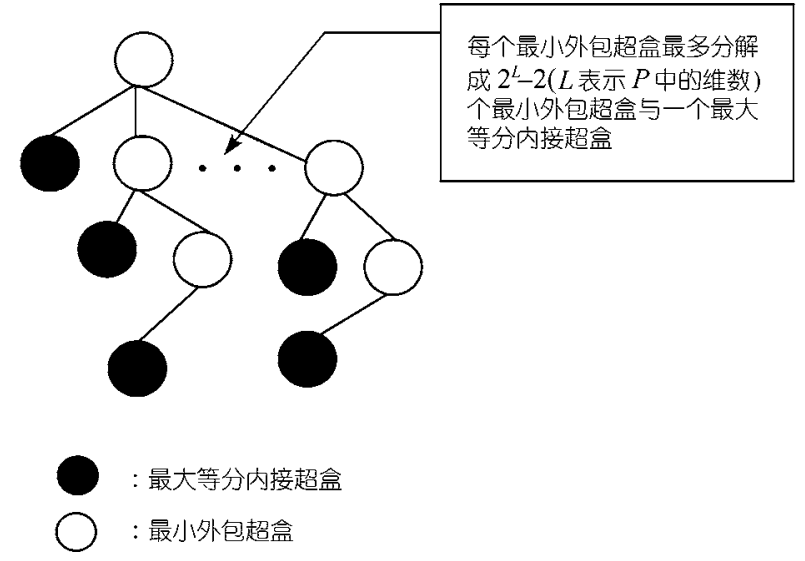

图 2 超盒组织的多叉树结构

多叉树就是基于偏序包含关系的堆结构. 图中黑圈表示最大等分内接超盒, 白圈 表示最小外包超盒. 很显然, 单调指标需求轨迹做交运算时, 如果某个超盒与多 叉树中的某个最小外包超盒不相交, 则其与此最小外包超盒内的所有子结点都 不相交, 这样在搜索求交时, 就可以剪枝处理, 大大减小计算量.

\section{2 逼近法}

如果对于任何一个指标, 所有使命需求对其的约束方向一致, 则可以采取类 似上一节中的逼近方法求出交区域. 其基本步骤与上一节单调指标需求轨迹生 成算法非常类似. 其主要思路如下:

1 把所有效需求轨迹的最小外包超盒求交，得出的超盒即为交区域的最小 外包超盒.

2 利用二分技术逐步逼近, 求出满足各个使命需求的最大等分内接超盒.

3 与上节算法一致, 逐步递归求解.

这种方法计算速度快, 适合并行处理, 但要求一致性, 不过大部分实际情况 都满足这一条件, 其实用价值较大.

图 3 解释了上述算法过程.

\section{3 优化蒙特卡罗算法}

这种算法是一种半解析, 半数值的方法, 它主要用于单调指标需求轨迹交区 域的数值积分计算. 它的计算量与超盒直接求交法正相反，即一般情况下求交的 单调指标需求轨迹个数越多, 它的计算量越小. 这种方法的基本思路是, 根据射 影几何的原理, 把 $n$ 维空间中的单调指标需求轨迹投影到 $n-1$ 个二维平面中, 然 后采用二维图形的求交方法, 求出各个单调指标需求轨迹在 $n-1$ 个二维平面投影 


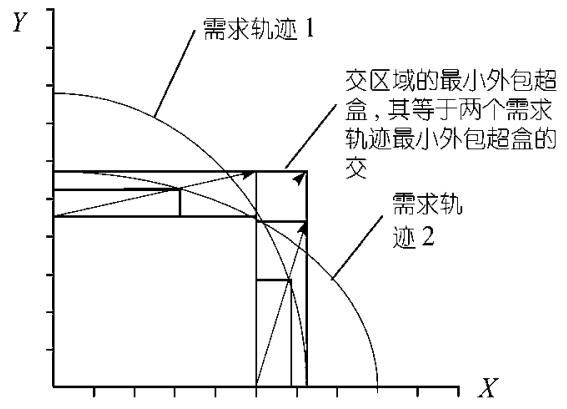

(a)

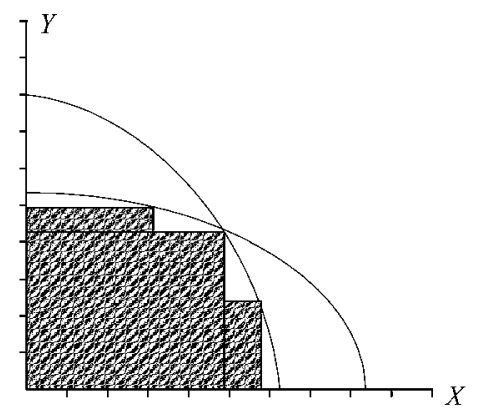

(b)

图 3 逼近法求单调指标需求轨迹交集的示意图

的交集，最后在经过大大缩小的空间区域中用蒙特卡罗方法进行积分计算. 由于 算法非常复杂, 具体内容可参看 ${ }^{1}$.

以上介绍三种单调指标需求轨迹求交的方法, 它们都有各自的优缺点以及 适用范围. 直接超盒求交法的优点是实现容易, 并能够切实的求出交的区域, 为 后续分析奠定了很好的基础, 但其缺点就是计算量较大. 逼近法计算速度快, 并 能够切实的求出交的区域, 但其必须要求指标需求的一致性. 对于优化蒙特卡罗 算法, 其优点是求交的单调指标需求轨迹数越多, 其计算量反而越小. 但其缺点 也是明显的，它不能够切实的求出交的区域，其只适合于交区域的积分计算. 因 此应该根据实际情况来做选择.

\section{6 基于单调指标空间的系统效能分析模型}

前文介绍了单个单调指标需求轨迹的生成与多个单调指标需求轨迹求交以 生成总单调指标需求轨迹的算法. 本节在此基础上研究了相应的效能分析模型.

\section{1 评估模型}

进行系统评估时, 必须明确需求, 以需求为对照, 以需求满足度作为有效性 评估的尺度. 由于复杂系统具有不确定性, 我们很难精确地确定系统的各个指标, 通常指标都是以概率分布形式描述的, 指标可以表示成 $p_{i}=p_{i e}+p_{i r}$, 其中 $p_{i e}$ 表示 指标 $p_{i}$ 的均值, 而 $p_{i r}$ 是一个随机变化量, 它通常可用一个概率密度函数来描述系 统的指标, 例如正态分布, 均匀分布等等. 我们用一联合概率密度函数来描述系 统指标, 设为 $f(p)$, 其中 $p$ 为单调指标空间的向量, 每一维代表一个效能指标值. 它可以通过数理统计的方法获得. 因此, 我们可以定义系统有效性值： $E=$

1) 同 359 页脚注 
$\int_{P l} f(p) \mathrm{d} p$, 其中 $P l$ 如前文所述的单调指标需求轨迹或总单调指标需求轨迹, 显 然 $0 \leqslant E \leqslant 1 . E$ 的实质上就是期望效用, 也就是预期一个系统满足一组特定任务要 求程度的量度. 如果单调指标需求轨迹是由超盒逼近而成, 则 $E \approx \sum_{j=1}^{N} \int_{H_{j}} f(p) \mathrm{d} p$, $H_{j}$ 表示为第 $j$ 个超盒, $N$ 表示超盒的总数, $P l \approx \bigcup_{j=1}^{N} H_{j}$.

如果使命描述是模糊化的，那么我们建立基于模糊单调指标需求轨迹的系 统模糊有效性计算公式:

$$
E_{f}=(F u z z y) \int_{p l} f(p) \mathrm{d} p=\underset{0 \leqslant \lambda \leqslant 1}{\vee}\left(\lambda \wedge \int_{f p l(\lambda)} f(p) \mathrm{d} p\right) .
$$

因为当 $\lambda_{1} \leqslant \lambda_{2}$ 时, $f p l\left(\lambda_{1}\right) \supseteq f p l\left(\lambda_{2}\right) ; \int_{f p l\left(\lambda_{1}\right)} f(p) \mathrm{d} p \geqslant \int_{f p l\left(\lambda_{2}\right)} f(p) \mathrm{d} p$. 如图 4 所示, 所以 $E_{f}=\lambda^{*}=\int_{f p l\left(\lambda^{*}\right)} f(p) \mathrm{d} p$, 用二分法或其他逼近方法快速求出 $E_{f \cdot}$. 模糊有效性 的计算不仅适合于实际应用中使命的模糊表示，而且还可以有效的抑制数据误 差带来的系统有效性的失真, 尤其当实际系统的效能(性能)指标集中分布在单调 指标需求轨迹的临界区域.

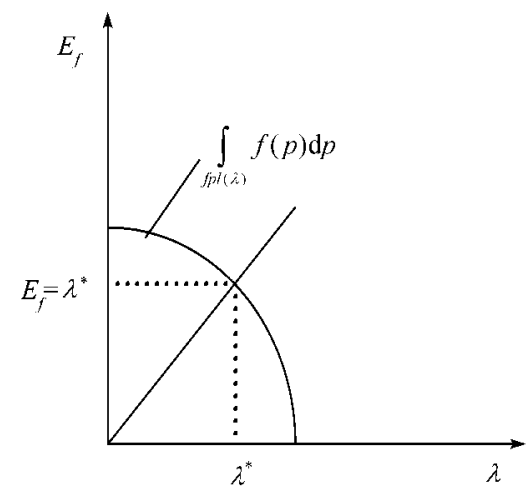

图 4 模糊积分的计算示意图

\section{2 指标的灵敏度分析}

在系统的效能分析过程, 我们往往需要 了解各个指标的灵敏度问题, 进行灵敏度分 析的目的有三:

1. 了解当前各个指标的相对重要性. 即 哪个指标的改善, 对系统总的有效性的改善 更敏感, 抓住主要环节.

2. 对难于肯定的环境条件, 或没有把握 确定的指标进行灵敏度分析，便于对那些敏 感的条件采取更加谨慎的处理.

3. 作为系统效能优化的基础.

灵敏度分析一般采用摄动法, 即某条件作微小变动后, 计算问题的输出结果 的相应变化量. 有条件时亦可采用解析法求解. 本文就是采用解析法求出指标的 灵敏度. 另外由于指标取值范围不一样, 通常在灵敏度分析这前, 应进行规格化 到相同的区间内，消除量纲，便于参照分析. 
定义 7 指标 $p_{i}$ 的灵敏度定义为

$$
S_{i}=\frac{\partial E}{\partial e_{i}}=\frac{\partial\left(\int_{P l} f(p, e) \mathrm{d} p\right)}{\partial e_{i}} \approx \frac{\partial\left(\sum_{j=1}^{N} \int_{H_{j}} f(p, e) \mathrm{d} p\right)}{\partial e_{i}},
$$

$p l \approx \bigcup_{j=1}^{N} H_{j} . e_{i}$ 是实际系统指标 $p_{i}$ 的期望值, 它是所要摄动分析的自变量.

从定义 7 可以看出, 指标的灵敏度是与需求和实际系统的表现是紧密相关 的, 其通常是一连续变化的量, 而不是一个静态值, 这样体现复杂非线性系统的 特点.

直接用上述定义求指标灵敏度是很不方便的, 而且计算的稳定性也很差, 其 实用性不佳.一般情况下, 实际系统的各个指标具有较好的独立性, 例如, 在一 防空系统中, 系统反应速度指标与稳定跟踪指数指标几乎完全独立. 因此, 我们 可以用如下更简洁的式子求指标的灵敏度，如定理 3.

定理 3 若各个指标统计独立, 则 $S_{i} \approx \sum_{j=1}^{N} T_{j, \bar{i}} \cdot\left(f_{i}\left(a_{j, i}\right)-f_{i}\left(b_{j, i}\right)\right)$, 其中 $N$ 是 超盒的个数, $f_{i}$ 是第 $i$ 个指标边缘密度函数, $b_{j, i}, a_{j, i}$ 是第 $j$ 个超盒中第 $i$ 个指标的 上下限, $T_{j, \bar{i}}=\prod_{1 \leq k \leq M, k \neq i} \int_{a_{j, k}}^{b_{j, k}} f_{k}\left(p_{k}, e_{k}\right) \mathrm{d} p_{k}, M$ 是指标的个数.

证 因为系统的各个指标统计独立, 所以

$$
\begin{aligned}
\int_{H_{j}} f(p, \mathrm{e}) \mathrm{d} p & =\int_{H_{j}} f_{1}\left(p_{1}, e_{1}\right) \cdot f_{2}\left(p_{2}, e_{2}\right) \cdots f_{M}\left(p_{M}, e_{M}\right) \mathrm{d} p_{1} \cdot \mathrm{d} p_{2} \cdots \mathrm{d} p_{M} \\
& =\left(\prod_{1 \leqslant k \leqslant M, k \neq i} \int_{a_{j, k}}^{b_{j, k}} f_{k}\left(p_{k}, e_{k}\right) d p_{k}\right) \cdot \int_{a_{j, i}}^{b_{j, i}} f_{i}\left(p_{i}, e_{i}\right) d p_{i}=T_{j, i} \int_{a_{j, i}}^{b_{j, i}} f_{i}\left(p_{i}, e_{i}\right) d p_{i} .
\end{aligned}
$$

把概率密度函数期望值作参变量平移扰动分析, 相当于对积分区间平移作 扰动分析.

$$
\begin{aligned}
\frac{d\left(\int_{b}^{a} f\left(p_{i}, e_{i}\right) \mathrm{d} p_{i}\right)}{d e_{i}} & =\lim _{\Delta e \rightarrow 0} \frac{\int_{b}^{a} f\left(p_{i}, e_{i}+\Delta e\right) \mathrm{d} p_{i}-\int_{b}^{a} f\left(p_{i}, e_{i}\right) \mathrm{d} p_{i}}{\Delta e} \\
& =\lim _{\Delta x \rightarrow 0} \frac{\int_{b-\Delta x}^{a-\Delta x} f\left(p_{i}, e_{i}\right) \mathrm{d} p_{i}-\int_{b}^{a} f\left(p_{i}, e_{i}\right) \mathrm{d} p_{i}}{\Delta x},
\end{aligned}
$$




$$
\frac{\partial\left(\int_{H_{j}} f(p, e) \mathrm{d} p\right)}{\partial e_{i}}=\lim _{\Delta x \rightarrow 0} \frac{T_{j, \bar{i}}\left(\int_{a_{j, i}-\Delta x}^{b_{j, i}-\Delta x} f_{i}\left(p_{i}, e_{i}\right) d p_{i}-\int_{a_{j, i}}^{b_{j, i}} f_{i}\left(p_{i}, e_{i}\right) \mathrm{d} p_{i}\right)}{\Delta x}
$$

$=\lim _{\Delta x \rightarrow 0} T_{j, i} \frac{\int_{a_{j, i}-\Delta x}^{a_{j, i}} f_{i}\left(p_{i}, e_{i}\right) \mathrm{d} p_{i}-\int_{b_{j, i}-\Delta x}^{b_{j, i}} f_{i}\left(p_{i}, e_{i}\right) d p_{i}+\int_{a_{j, i}}^{b_{j, i}} f_{i}\left(p_{i}, e_{i}\right) d p_{i}-\int_{a_{j, i}}^{b_{j, i}} f_{i}\left(p_{i}, e_{i}\right) \mathrm{d} p_{i}}{\Delta x}$

$=T_{j, \bar{i}} \cdot\left(f\left(a_{j, i}\right)-f\left(b_{j, i}\right)\right)$

则 $\frac{\partial\left(\sum_{j=1}^{N} \int_{H_{j}} f(p, e) \mathrm{d} p\right)}{\partial e_{i}}=\sum_{j=1}^{N} T_{j, \bar{i}} \cdot\left(f\left(a_{j, i}\right)-f\left(b_{j, i}\right)\right)$. 证毕.

根据定理 3, 求指标灵敏度的时候, 就可避开求导, 直接用积分值计算. 因 而, 对于数值算法来说其计算精度与稳定性都有很大的提高.

\section{3 指标的优化分配设计}

系统指标的优化设计也是广义效能分析中的一个重要内容. 此问题也就是 如何以最小的代价(时间、金钱等)优化设计系统的各项指标, 以使系统能够达到 用户的需求. 这样, 我们就可以很自然的在单调指标需求轨迹的基础上建立一个 条件优化模型, 进行优化解算. 具体的实现方法可参看 ${ }^{1)}$.

\section{7 案例：对一反隐身防空信息系统的效能分析}

\section{1 基本想定}

本案例是分析了一种反隐身防空信息系统方案的有效性. 其基本想定如下: 根据隐身飞机在两侧隐身效果差的缺陷，把防空导弹隐蔽部署在敌来袭飞机空 中走廊的两侧. 远程概略警戒发现目标后, 它把目标信息传送到指挥中心, 指挥 中心进行情报处理, 目标分析等等工作, 然后向隐蔽部署的防空导弹阵地发出开 机射击命令, 并指示目标方位. 击落敌机后, 各导弹阵地迅速隐蔽撤离, 以免被 摧毁.

在本例中假定通过某种情报手段得知敌隐身飞机将通过图 5 所指的空中走 廊, 攻击我方目标 $T$. 我方根据隐身飞机侧方的隐身效果较差的原理, 以敌机航 线为 $X$ 轴建立坐标系, 在空中走廊两侧部署了一部远程警戒雷达 $R_{1}(30,-50)$, 四 个防空导弹阵地 $\left(M_{1}-M_{4}\right)$, 它们的坐标分别是: $(50,-20),(60,-35),(65,30),(75$, 20). 指控中心 $C$ 负责数据融合与指示目标. 由于隐身飞机的雷达反射面积 $(R C S)$

1) 同 359 页脚注 


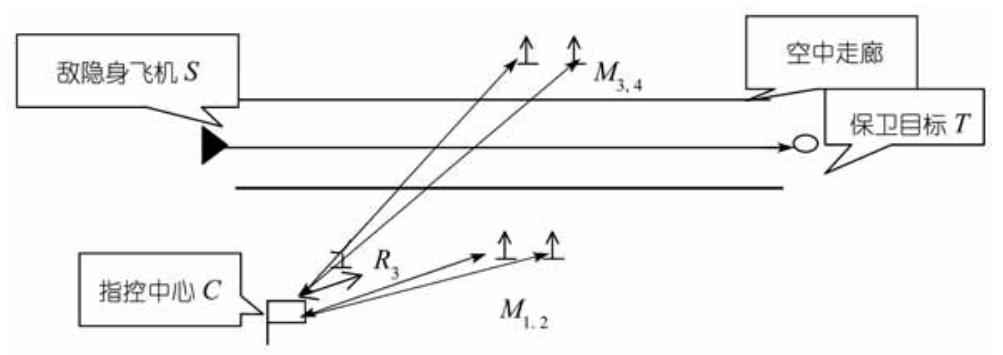

图 5 一个反隐身防空信息系统的作战概念图

较小, 且难以连续掌握目标, 所以雷达制导的防空导弹有效射击窗口很窄, 战机 稍纵即逝, 因此对系统效能(性能)指标提出了更高的要求. 假设我将以 $P \geqslant 0.7$ 概 率将敌机击落为使命需求约束条件. 本例主要是以此为需求, 得出相应的系统关 键指标的需求轨迹, 以进行效能分析, 对其他相关想定参数假定已知. 系统关键 指标是:

远程警戒雷达的探测性能指标 $K_{1}$ : 在防空作战中, 远程警戒雷达负责尽远的 发现目标, 然后传送到指控中心进行分析处理. $K_{1}$ 是一个由发射功率, 增益系数 等多个指标计算合成的一个描述雷达探测能力的综合指标. 它与敌机雷达反射 面积共同确定了最远发现距离. 很显然它相对于使命需求指标(击落敌机概率)是 一个单调增的指标.

系统处理延迟 $T$ : 它表示远程警戒雷达发现目标后上报到指挥所, 经指挥所 处理, 然后指示给各导弹阵地所需时间, 它也就是通信时延与决策处理时延合成 的一个综合指标. 由于战机稍纵即逝, 因此延迟必须短, 它是单调减指标.

跟踪雷达性能指标 $K_{2}$ : 它是衡量导弹制导雷达能否有效跟踪敌机的性能指 标, 同样它与目标雷达反射面积一道决定了稳定跟踪概率. 它是单调增的指标.

以上三个综合指标是决定能否完成系统使命的主要因素, 它们都是单调类 型的指标. 为了描述方便, 本文把这几个指标的取值范围规范化到 $[0,100]$ 区间内.

另外, 敌来袭飞机可能是 $\mathrm{A}$ 型或 $\mathrm{B}$ 型隐身飞机，它们的主要参数如下:

A 型隐身飞机其空防速度为: $1000 \mathrm{~km} / \mathrm{h}$, 其各个角度上的雷达反射面积 $(R C S)$ 如下表:

\begin{tabular}{cccccccccccccc}
\hline 角度 & 10 & 20 & 30 & 40 & 50 & 60 & 70 & 80 & 90 & 100 & 110 & 120 & 130 \\
\hline $\begin{array}{c}\text { RCS } \\
/ \mathrm{m}^{2}\end{array}$ & 0.01 & 0.02 & 0.05 & 0.06 & 0.1 & 0.2 & 0.5 & 0.9 & 1 & 0.8 & 0.7 & 0.5 & 0.4 \\
140 & 150 & 160 & 170 & 180 & & & & & & & & & \\
0.2 & 0.15 & 0.08 & 0.05 & 0.02 & & & & & & & \\
\hline \multicolumn{7}{c}{ B 刑隐身飞机其主要参数是. 各个角度上的雷达反射面和都近似于 $4 \mathrm{~m}^{2}$ 其突防速度为 $1500 \mathrm{~km} / \mathrm{h} ;$}
\end{tabular}

\section{2 效能分析}

系统的根本需求就是要击落敌隐身飞机, 即按照军语的解释即为以大于 0.7 
的概率击落敌隐身飞机. 对于能否实现这一作战需求，其关键就是上述三个效能 指标取值情况，以及相应的作战环境. 在本例中, 要想建立系统指标与使命需求 的之间显式的解析函数关系是非常困难. 因此, 只能通过仿真与查数据表的方法 来建立这种关系. 利用前述方法与仿真手段求出相应的单调指标需求轨迹如图 6 所示(为了显示方便, 分别用 $100-K_{1}, 100-K_{2}$ 来代替 $K_{1}, K_{2}$ ):

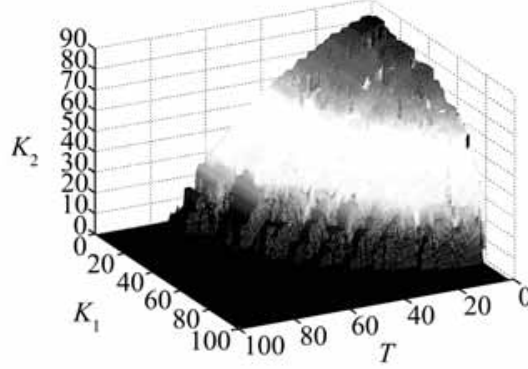

(a)

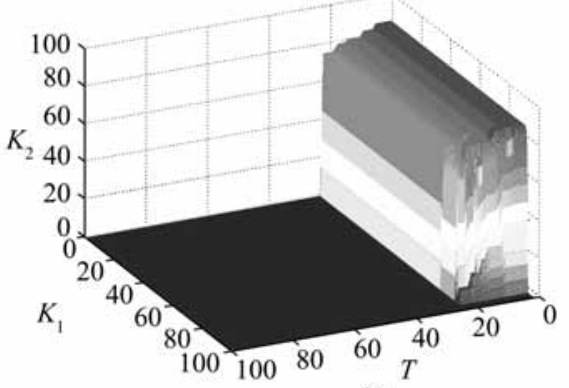

(b)

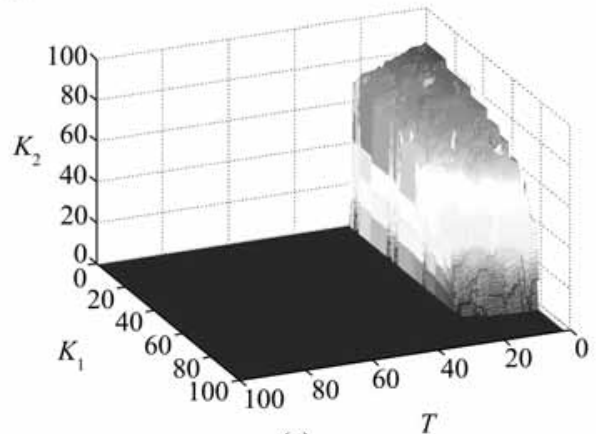

(c)

图 6

(a) 对于 $\mathrm{A}$ 型隐身飞机的单调指标需求轨迹; (b) 对于 B 型隐身飞机的单调指标需求轨迹; (c) 利用 5.2 中逼近法求出的考虑两种使命需求的总单调指标需求轨迹

若某一系统的它们指标分布如下:

$$
K_{1}=\frac{1}{\sqrt{(2 \pi) 2}} e^{\frac{-\left(K_{1}-45\right)^{2}}{8}}, T=\frac{1}{\sqrt{(2 \pi) 5}} e^{\frac{-(T-20)^{2}}{50}}, K_{2}=\frac{1}{\sqrt{(2 \pi) 2}} e^{\frac{-\left(K_{2}-50\right)^{2}}{8}}
$$

当突防的是 $\mathrm{A}$ 型隐身飞机(其轨迹如图 6(a))则防空信息系统的有效性 $E=0.88$, 利用定理 3 计算出的三个指标的灵敏度为: $(0.03,-0.28,0.15)$, 三个指标都较为灵 敏. 当突防的是 B 型隐身飞机(其轨迹如图 6(b)则防空信息系统的有效性 $E=0.73$, 三个指标的灵敏度为: $(0.00001,-0.06,0.0000001)$, 很显然由于敌机雷达反射面积 较大, 雷达探测, 跟踪性能指标的灵敏度很小, 即不是重要指标; 若以总需求作 为评价尺度(其轨迹如图 6(c)), 则其效性 $E=0.7$. 


\section{参考文献}

1 兵器工业部兵器系统工程研究所译. 陆军武器系统分析. 北京: 兵器工业出版社, 1985. 403 415

2 魏世孝, 周献中. 多属性决策理论方法及其在 C3I 系统中的应用. 北京: 国防工业出版社, 1998. $86 \sim 15$

3 Bouthonnierand L. Effectiveness analysis of C3 systems. IEEE Transaction on Systems, Man and Cybernetics, 1984, 14(1): 48 54

4 Christine M. Bther. Computer Graphics for System Effectiveness Analysis. AD-A 173546

5 John F S. Command and (Out of) Control: The Military Implications of Complexity Theory. the Complexity. Global Politics and National Security Conference, 1996. 13 14

6 胡剑文, 张维明, 刘 忠. 复杂系统理论及其对作战实践的指导研究. 火力与指挥控制, 2004, 29(2): 68 71

7 Moose P H. Evolution Equations of C3I: Cannonical Forms and Their Properties. ADA135293

8 Moss L. An Analysis of the MCES:Application to the IFFN Joint Testbed. AD-A181

9 吴晓锋. SEA 方法及其在 C3I 系统效能分析中的应用(4)一一效能分析. 系统工程理论与实践, 1999(2): 44 49 